\title{
Evolution of Star Clusters in Time-variable Tidal Fields
}

\author{
Ernest N. Mamikonyan ${ }^{1}$, Stephen L. W. McMillan ${ }^{1}$, Enrico Vesperini ${ }^{2}$, and Mordecai-Mark Mac Low ${ }^{1,3,4}$ \\ ${ }^{1}$ Department of Physics, Drexel University, Philadelphia, PA 19104, USA; ernest@physics.drexel.edu, steve@physics.drexel.edu \\ 2 Astronomy Department, Indiana University, Bloomington, IN 47405, USA; evesperi@indiana.edu \\ ${ }^{3}$ Department of Astrophysics, American Museum of Natural History, New York, NY 10024, USA; mordecai@amnh.org \\ ${ }^{4}$ Institut für Theoretische Astrophysik, Zentrum für Astronomie der Universität Heidelberg, D-69121 Heidelberg, Germany \\ Received 2014 December 10; revised 2016 November 17; accepted 2017 January 29; published 2017 March 3
}

\begin{abstract}
Strong tidal forces can dominate star cluster evolution in merging galaxies, determining their mass-loss rates and lifetimes. In order to model this evolution, we have developed a second-order accurate numerical method for integrating a star cluster in an arbitrary time-variable tidal field. We extend the KIRA $N$-body integrator to handle these external fields. We obtain realistic tidal histories from a galaxy merger simulation including sink particles, which we interpret as young star clusters. Coupling these tidal accelerations to $N$-body models of isolated clusters, we perform detailed dynamical studies. This generalizes the formalism previously used to explore the dynamical effects of the galactic tidal field on clusters in circular orbits. We find that, in contrast to previous studies that considered only stellar and dark matter dynamics, tidal interactions between clusters and dense gas in the galactic disk can significantly influence cluster mass loss and lifetimes. Using our models, we develop an effective semianalytic model that can be used for fast estimation of cluster mass loss in a galactic tidal field and to study the evolution of the globular cluster mass function in isolated and merging galaxies.
\end{abstract}

Key words: galaxies: interactions - galaxies: star clusters: general - methods: numerical

\section{Introduction}

The dynamical evolution of star clusters can be significantly affected by the external tidal field of their host galaxy. The effects of simple galactic tidal fields, such as those from a point-mass or isothermal halo, on clusters moving on regular orbits have been extensively studied both analytically and numerically. Studies of the effect of external galactic fields include the definition of new analytical equilibrium models based on distribution functions depending on the Jacobi integral (Heggie \& Ramamani 1995; Bertin \& Varri 2008; Varri \& Bertin 2009) and extensive analytical and numerical studies of the effects of the external tidal field on the cluster dynamical evolution for clusters on circular and eccentric orbits, including the effects of tidal shocks occurring when clusters cross a galactic disk (e.g., Chernoff et al. 1986; Spitzer 1987; Weinberg 1994a, 1994b, 1994c; Vesperini \& Heggie 1997; Baumgardt \& Makino 2003, see also Heggie \& Hut 2003 and references therein).

Although these studies have shed light on many fundamental aspects of the role of external tidal fields on the properties and evolution of globular clusters, their relevance is limited to clusters evolving in galaxies in equilibrium. In many circumstances, however, these approximations are clearly not valid. Of particular interest are the rapidly varying tidal fields found during galaxy mergers, of which the Antennae galaxies (Whitmore et al. 1999) are perhaps the best-studied example. The central regions of the Antennae and other starburst systems are characterized by irregular density distributions and strong rapidly varying tidal fields. Newly formed clusters may find themselves on orbits that repeatedly pass through these irregular regions before they eventually become part of the general cluster population of the resulting merged galaxy. The strong time variation in tidal stresses during the merger may significantly influence the evolution of these young clusters, as well as that of older clusters already present in the merging galaxies.
The complex tidal field experienced by a cluster during a merger event or the early phases of galaxy assembly is difficult to model analytically, leading previous workers to adopt semianalytical (Kruijssen et al. 2012) or numerical methods (Renaud et al. 2011; Rieder et al. 2013). We follow their example by adopting the more practical approach of obtaining realistic tidal histories for stellar and cluster orbits from numerical simulations of galaxy mergers. Our data are drawn from a simulation described by Li et al. (2004, 2007), which describes a merger of two galaxies with masses similar to the Antennae, including an isothermal gas component, and also includes a prescription for sink particle formation ( $\mathrm{Li}$ et al. 2005) to model the formation of clusters during the merging process. Renaud \& Gieles (2013), who performed a similar analysis, did not include the effects of gas in their galaxy merger model.

Our goal in this paper is to study the evolution of clusters during a galactic merger. To do so, we have developed a second-order accurate numerical method to study the dynamical evolution of star clusters under arbitrary tidal fields with strong variations in both space and time. We extend the collisional integrator KIRA, which is part of the STARLAB package (Portegies Zwart et al. 2001), to include this method, and then apply it to the problem at hand.

By computing the tidal acceleration tensor along the trajectory of every sink particle, we obtain the full tidal history of every star cluster formed during the merger process. We then select orbits of interest and perform detailed dynamical simulations of star clusters using these tidal histories. We present several such simulations in this paper. We use them to derive a reasonably accurate semianalytic approximation of cluster mass loss in the presence of an arbitrary time-variable tidal field.

\section{Approximating Tidal Effects}

We seek to estimate the tidal effects of the galactic environment on the internal dynamics of a star cluster. It is 
standard practice (e.g., Hartle 2003) to define a tidal acceleration tensor,

$$
T_{i j} \equiv-\partial_{i} \partial_{j} \Phi
$$

where $\Phi(\boldsymbol{x})$ is the gravitational potential of the surrounding galaxy at the cluster center of mass $\boldsymbol{x},{ }^{5}$ and $\partial_{i} \equiv \partial / \partial x_{i}$.

For a galactic system of inertial coordinates containing $N$ discrete particles with masses $m_{k}$ and positions $\boldsymbol{x}_{k}(k=$ $1, \ldots, N)$, the potential at the cluster center is

$$
\Phi(\boldsymbol{x})=-\sum_{k=1}^{N} \frac{G m_{k}}{r_{k}}
$$

where $r_{k}=\left|\boldsymbol{x}-\boldsymbol{x}_{k}\right|$. (Note that in practice we implement a softened version of this potential as discussed below in Section 3.1.) Hence the acceleration is

$$
a_{j}=-\partial_{j} \Phi=-\sum_{k=1}^{N} \frac{G m_{k}\left(x_{j k}-x_{j}\right)}{r_{k}^{3}}
$$

where $x_{j k}$ is the $j$ th component of $\boldsymbol{x}_{k}$, and

$$
T_{i j}=\partial_{i} a_{j}=\sum_{k=1}^{N} G m_{k}\left[\frac{\left(x_{i k}-x_{i}\right)\left(x_{j k}-x_{i}\right)}{r_{k}^{5}}-\frac{\delta_{i j}}{r_{k}^{3}}\right] .
$$

This tensor is symmetric with six independent components that can be derived from an arbitrary mass distribution. In this paper, we take that distribution from large-scale numerical models. This is the same approach used by Renaud et al. (2011). We note that Poisson's equation,

$$
\nabla^{2} \Phi=4 \pi G \rho,
$$

implies that the trace of the tidal acceleration tensor is related to the local density by

$$
\sum_{i} T_{i i}=-4 \pi G \rho
$$

We expect the reference frame of the cluster (in typical galactic environments) to be approximately inertial. This is confirmed by the top two panels in Figure 8 of Renaud et al. (2011), which show that tidal forces far exceed the centrifugal contribution.

Coriolis forces can be neglected in our study because even though the galaxy dominates the orbital motion, the primary tidal forces are not exerted by the large-scale potential of the galaxy, but rather by nearby small-scale density perturbations in our model (likely giant molecular clouds in reality). To examine the importance of Coriolis forces in more detail, consider a cluster orbiting at distance $R_{\mathrm{G}}$ in a galaxy of mass $M_{\mathrm{G}}$, with a closer perturber of mass $m^{\prime}$ at distance $r^{\prime}$, which will be several times the tidal radius $r_{\mathrm{t}} \sim 50 \mathrm{pc}$ for cases of interest.

The center-of-mass orbital acceleration of the cluster due to the galaxy is $a_{\mathrm{g}}=G M_{\mathrm{G}} / R_{\mathrm{G}}^{2}$, while that for the perturber is

\footnotetext{
5 Here, we are only concerned with the external potential; the self-gravity of the star cluster is already included in the $N$-body code we use and is not included here.
}

$a_{\mathrm{p}}=G m^{\prime} / r^{\prime 2}$, so their ratio is

$$
\begin{aligned}
\frac{a_{\mathrm{p}}}{a_{\mathrm{g}}} & \sim\left(\frac{m^{\prime}}{M_{\mathrm{G}}}\right)\left(\frac{r^{\prime}}{R_{\mathrm{G}}}\right)^{-2} \\
& =0.1\left(\frac{m^{\prime}}{10^{6} M_{\odot}}\right)\left(\frac{M_{\mathrm{G}}}{10^{11} M_{\odot}}\right)^{-1}\left(\frac{r^{\prime}}{100 \mathrm{pc}}\right)^{-2}\left(\frac{R_{\mathrm{G}}}{10 \mathrm{kpc}}\right)^{2},
\end{aligned}
$$

suggesting that for these typical parameters the galaxy indeed dominates the center-of-mass motion. On the other hand, the tidal accelerations of a star at distance $r$ from the center of the cluster due to the galaxy is $a_{\mathrm{tg}}=2 G M_{\mathrm{G}} r / R_{\mathrm{G}}^{3}$, while that for the perturber is $a_{\mathrm{tp}}=2 G m^{\prime} r / r^{\prime 3}$, so their ratio is

$$
\begin{aligned}
\frac{a_{\mathrm{tp}}}{a_{\mathrm{tg}}} & \sim\left(\frac{m^{\prime}}{M_{\mathrm{G}}}\right)\left(\frac{r^{\prime}}{R_{\mathrm{G}}}\right)^{-3} \\
& =10\left(\frac{m^{\prime}}{10^{6} M_{\odot}}\right)\left(\frac{M_{\mathrm{G}}}{10^{11} M_{\odot}}\right)^{-1}\left(\frac{r^{\prime}}{100 \mathrm{pc}}\right)^{-3}\left(\frac{R_{\mathrm{G}}}{10 \mathrm{kpc}}\right)^{3},
\end{aligned}
$$

which means that the perturber dominates the tidal field. Now, how does the Coriolis force compare to the dominant tidal force from the perturber? The effective Coriolis acceleration on a star moving at speed $v$ relative to the cluster center is $a_{\mathrm{c}} \sim \Omega v$, where $\Omega^{2} \sim G M_{\mathrm{G}} / R_{\mathrm{G}}^{3}$. Hence $a_{\mathrm{c}} \sim a_{\mathrm{g}}\left(v / V_{\mathrm{G}}\right)$, where $V_{\mathrm{G}}$ is the orbital speed of the cluster in the galactic potential. Comparing this Coriolis acceleration with the tidal acceleration due to the perturber at distance $r^{\prime}$ from the cluster center yields

$$
\begin{aligned}
\frac{a_{\mathrm{c}}}{a_{\mathrm{tp}}} \sim & 0.5\left(\frac{m^{\prime}}{M_{\mathrm{G}}}\right)^{-1}\left(\frac{r^{\prime}}{R_{\mathrm{G}}}\right)^{2}\left(\frac{v}{V_{\mathrm{G}}}\right)\left(\frac{r^{\prime}}{r}\right) \\
= & 0.05\left(\frac{m^{\prime}}{10^{6} M_{\odot}}\right)^{-1}\left(\frac{M_{\mathrm{G}}}{10^{11} M_{\odot}}\right) \\
& \times\left(\frac{r^{\prime}}{100 \mathrm{pc}}\right)^{3}\left(\frac{R_{\mathrm{G}}}{10 \mathrm{kpc}}\right)^{-2} \\
& \times\left(\frac{v}{1 \mathrm{~km} \mathrm{~s}^{-1}}\right)\left(\frac{V_{\mathrm{G}}}{200 \mathrm{~km} \mathrm{~s}^{-1}}\right)^{-1}\left(\frac{r}{50 \mathrm{pc}}\right)^{-1},
\end{aligned}
$$

where we have chosen $r=r_{\mathrm{t}}$ for our scaling. The ratio is greatest near the cluster tidal radius, although this effect is somewhat mitigated by the slower orbital speeds there. We conclude that the tidal force from a perturber is likely to significantly exceed the Coriolis forces near the boundaries of a cluster where stars can be lost, but that future work would be justified in considering this problem more carefully.

Rieder et al. (2013) also neglect non-inertial terms. In this frame, linearized equations of motion for each star's potential, acceleration, and jerk are (Spitzer 1987; Heggie \& Ramamani 1995; Renaud et al. 2011)

$$
\begin{gathered}
\phi=-\frac{1}{2} \sum_{i, j} T_{i j} \xi_{i} \xi_{j}, \\
a_{i}=\sum_{j} T_{i j} \xi_{j},
\end{gathered}
$$




$$
j_{i} \equiv \frac{d a_{i}}{d t}=\sum_{j}\left[\frac{d T_{i j}}{d t} \xi_{j}+T_{i j} u_{j}\right]
$$

where $\xi_{i}$ and $u_{i}$ are positions and velocities, respectively, relative to the cluster center of mass. We include the jerk here because it is required in the fourth-order integration scheme that we use, as mentioned in Section 3. As usual, this approximation is valid for $\xi \ll x$.

In order to estimate the Jacobi radius and construct a semianalytic model for mass loss, it is sometimes convenient to consider the diagonalized form of the tidal tensor, whose eigenvalues we denote $\alpha_{i}$. We can always do this by momentarily constructing a coordinate system at the location of the cluster such that the tidal acceleration is aligned along its axes. The sign convention of Equation (4) is such that a positive eigenvalue implies an extensive tide along the direction of its eigenvector, and a negative eigenvalue implies a compressive tide. Furthermore, since we consider tidal disruption, we sometimes focus on the highest eigenvalue $\alpha_{1}$, which signifies the strength of the dominant mode of mass loss.

\section{Stellar Dynamics}

To follow the dynamical evolution of the star cluster population, we carry out a number of $N$-body simulations using the KIRA code. KIRA is direct-summation, block timestep (McMillan 1986; Makino 1991b) integrator based on the fourth-order Hermite scheme (Makino 1991a). It is part of the extensive and well-tested STARLAB (Portegies Zwart et al. 2001) environment for exploring the dynamical and stellar evolution of star clusters and other dense systems. KIRA has a variety of back ends, which can offload the $\mathcal{O}\left(N^{2}\right)$ portion of the calculation onto a Graphics Processing Unit (GPU) via the SAPPORO library (Gaburov et al. 2009), several variants of GRAPE hardware (Makino et al. 2003), or fall back to the CPU. For performance reasons, we used the GPU-accelerated version on a cluster with 120 NVIDIA Fermi GPUs.

Previously, KIRA only had the ability to evolve systems in an external tidal field chosen from a predefined set of simple analytical distributions, e.g., point-mass or isothermal, as elaborated in Section 3.1. Furthermore, the implementation of Equations (10) and (11) included only two diagonal elements of $T_{i j}$. We extended this functionality to arbitrary non-diagonal time-dependent tidal tensors, such as those that arise during complex galactic encounters, by replacing analytic external potentials with those computed from Equation (4). We follow Renaud et al. (2011) in computing a tabulated time series of values of $T_{i j}$ from the larger galaxy merger simulation for use in the cluster simulations.

We handle the additional complication of a time-dependent tidal field drawn from a larger-scale model by sampling the larger model at regular time intervals and calculating the tidal field at those points in time. We then apply the field at the cluster position to the $N$-body simulation in a piecewise constant fashion, thus neglecting the associated time derivatives. This neglect is acceptable because the energy input at each discontinuity in the tidal forcing is small compared to the total energy, which we show is true for the models we use in Section 4.1 below.

We implement this method simply by halting and reinitializing at every change of the tidal field. This avoids the spurious higher derivatives that would cause numerical instability at the transitions in tidal force. With this procedure, the energy is conserved to the usual level found with KIRA of a few percent.

Stars are permanently removed from the simulation for computational convenience if they cross the $r=25 r_{\mathrm{v}}$ boundary, where $r_{\mathrm{v}}$ is the virial radius. We assume that the linearized tidal acceleration is still valid at this distance. However, the choice of radius is conservative and somewhat arbitrary. The large radius of the boundary was selected to try to ensure that we never eliminate stars that might otherwise return to the vicinity of the cluster. As long as these distant stars do not significantly affect the dynamics of the cluster, we think it is better to be less aggressive in removing them from the simulation, even if the approximate acceleration acting on them is less accurate out there. We have experimented with values between $15 r_{\mathrm{v}}$ and $35 r_{\mathrm{v}}$, and the particular cutoff radius chosen has an effect weaker than $2 \%$ on the total mass at any time in the evolution of the cluster, and weaker than $1 \%$ at late times.

In anticipation of later discussion, we define some relevant timescales here. The dynamical time at radius $r$ is

$$
t_{\mathrm{d}}(r)=\sqrt{\frac{r^{3}}{G M}} .
$$

We are primarily interested in the values at the half-mass radius $r_{\mathrm{h}}$ and the tidal (or Jacobi) radius $r_{\mathrm{t}}$, where

$$
r_{\mathrm{t}}^{3}=\frac{G M}{\alpha_{1}}
$$

which we denote $t_{\mathrm{dh}}$ and $t_{\mathrm{dt}}$,

$$
\begin{aligned}
& t_{\mathrm{dh}}=\sqrt{\frac{r_{\mathrm{h}}^{3}}{G M}} \\
& t_{\mathrm{dt}}=\sqrt{\frac{r_{\mathrm{t}}^{3}}{G M}},
\end{aligned}
$$

The half-mass relaxation time of a system of $N$ particles each of mass $m$ is (Spitzer 1987)

$$
t_{\mathrm{rh}}=\frac{0.138\left(N r_{\mathrm{h}}^{3}\right)^{1 / 2}}{(G m)^{1 / 2} \ln (\gamma N)},
$$

where we have adopted the Spitzer (1987) value $\gamma=0.4$ (but see Giersz \& Heggie 1994). We use the initial half-mass relaxation time as the time unit for all figures showing cluster structural evolution. The relaxation times at the abovementioned locations are

$$
\begin{aligned}
t_{\mathrm{rh}} & \propto N t_{\mathrm{dh}}, \\
t_{\mathrm{rt}} & \propto N t_{\mathrm{dt}},
\end{aligned}
$$

neglecting logarithmic terms.

However, the properties of the $N$-body system per se are quoted in terms of the dynamical time at the virial radius $t_{\mathrm{dv}}$ because the simulations are scaled in standard Hénon units, according to Heggie \& Mathieu (1986); these are described in detail in Section 5.

For all simulations, we used the initial Plummer distribution with $N_{\mathrm{p}}=16,384$ equal-mass particles, all of which are cluster members. This neglects any structure remaining from the initial formation of the cluster, but should be sufficient to capture the dynamical evolution of an equilibrium gas-free cluster over 


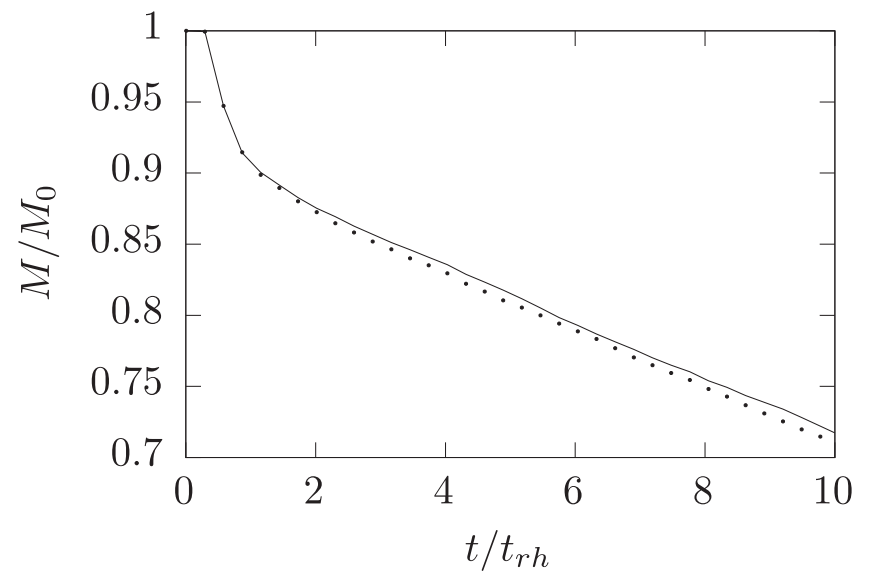

Figure 1. Mass evolution of a star cluster in a circular orbit around a pointmass potential. The dotted curve shows KIRA's original special-purpose implementation, and the solid curve shows the new generalization to an arbitrary tidal acceleration.

galactic dynamical timescales. The Plummer model,

$$
\rho(r)=\frac{3 M}{4 \pi b^{3}}\left(1+\frac{r^{2}}{b^{2}}\right)^{-5 / 2},
$$

where $b$ is a scale parameter, is an equilibrium density distribution commonly used in dynamical simulations. We have also considered King models with various concentration parameters, but could find no substantive impact on their later mass-loss histories, which is the quantity of most interest to us. The half-mass relaxation time with this many particles is

$$
t_{\mathrm{rh}} \approx 174 t_{\mathrm{dv}} \text {. }
$$

\subsection{Circular Orbit in Point-mass Potential}

As a test of our formalism and its implementation in KIRA, we compare the mass loss of a cluster on a circular orbit, including centrifugal effects, in a point-mass potential, using the original special-case implementation and the new general one. We use this particular test configuration because it is one of the built-in orbits in the prior version of Kira. This is not in the inertial frame, unlike the general case we considered in Section 2, or the numerical model we use for clusters in merging galaxies that we describe below. In this simple case the tidal acceleration tensor is constant, diagonal, and readily obtainable (Spitzer 1987):

$$
\boldsymbol{T}=\frac{G M_{\mathrm{G}}}{R_{\mathrm{G}}^{3}}\left[\begin{array}{lll}
3 & & \\
& 0 & \\
& & -1
\end{array}\right],
$$

where $M_{\mathrm{G}}$ is the mass of the point-mass producing the potential, and $R_{\mathrm{G}}$ is its distance.

The mass of a cluster in the field of Equation (22) is shown in Figure 1; the initial strength of the tidal field can be characterized by $\frac{r_{\mathrm{t}}}{r_{\mathrm{h}}}=5.2$. Mass loss commences after the cluster has expanded to fill the cutoff surface at $25 r_{\mathrm{v}}$. As expected, the tidal field induces mass loss in two phases: (a) mass outside the newly imposed tidal radius becomes unbound and is removed rapidly; and (b) the remaining stable star cluster undergoes relaxation, marked by linear mass loss. In Figure 1, the first phase lasts roughly $t_{\mathrm{rh}}$. In both this test and our production models, we run for approximately $10 \mathrm{t}_{\mathrm{rh}}$, or $1.7 \mathrm{Gyr}$, for our standard parameters. The agreement between the two versions is generally good. A small difference in the realization of the initial Plummer model for the $N$-body system can easily explain the remaining differences. We can eliminate any non-inertial terms as a cause of the difference since both tests were run with the same set of (non-inertial) assumptions.

The enhanced version of KIRA outputs and samples the tidal acceleration at fixed intervals and reinitializes the entire system whenever the value of the tidal acceleration changes. Obviously, this is not necessary in the case of the constant tidal field, but we applied this procedure to both versions of KIRA to maintain consistency with later simulations.

\section{Galactic Merger Simulations}

Tidal histories were obtained by post-processing results from the galaxy merger simulations of Li et al. (2004, 2007), which were carried out using version 1.1 of the open-source smoothed particle hydrodynamics code GADGET (Springel et al. 2001) extended with the sink particle prescription of $\mathrm{Li}$ et al. (2005). Sink particles were formed in regions of high gas density $\left(n>10^{3} \mathrm{~cm}^{-3}\right.$ ), replacing gravitationally bound gas particles while conserving mass and linear and angular momenta. The original sink particle formalism for smoothed particle hydrodynamics simulations on which it is based was described by Bate et al. (1995).

The simulations were initialized with bulgeless galactic disks, each containing a dark matter halo, exponential stellar disk, and gas with an isothermal equation of state, and sound speed $c_{\mathrm{s}}=6 \mathrm{~km} \mathrm{~s}^{-1}$. This value for the sound speed was chosen to reproduce the effects of stellar feedback on the dynamics of the gas, maintaining the star formation rate at a realistic value. This simple model ensures a converged star formation rate ( $\mathrm{Li}$ et al. 2005) that agrees with the SchmidtKennicutt law (Kennicutt 1998), since the details of the turbulence are approximated by the high sound speed. Although much more detailed treatments of the physics of the interstellar medium and star formation have since been developed, the relatively tractable models of Li et al. (2007) capture the basic dynamics of the tidal forcing. The particular model used for all tidal histories in this paper was referred to as MG2 in Li et al. (2007); its progenitor galaxy haloes had masses $3.3 \times 10^{11} M_{\odot}$ and rotation velocities $100 \mathrm{~km} \mathrm{~s}^{-1}$. Gas particles had masses of $6.6 \times 10^{3} M_{\odot}$, giving a mass resolution of $\sim 5 \times 10^{5} M_{\odot}$, and had softening lengths of $10 \mathrm{pc}$. The softening lengths of the dark matter halo particles were $0.4 \mathrm{kpc}$, while for the sink particles and stellar disk particles they were $0.1 \mathrm{kpc}$. Half of the $2 \times 10^{6}$ particles were gas, $30 \%$ were dark matter, and $20 \%$ were disk stars. The collisionless softening lengths substantially exceed a typical globular cluster size of $r_{\mathrm{h}} 10 \mathrm{pc}$. The masses of the galaxies in this model are similar to those of the Antennae merging galaxies (Whitmore \& Schweizer 1995), although the orbits are not the same.

This merger simulation produced full system output, including positions and velocities of all particles, at regular intervals of $10 \mathrm{Myr}$; these were used for all further analysis. This sampling time appears sufficiently frequent, as it is comparable to the dynamical time at the tidal radius for typical 


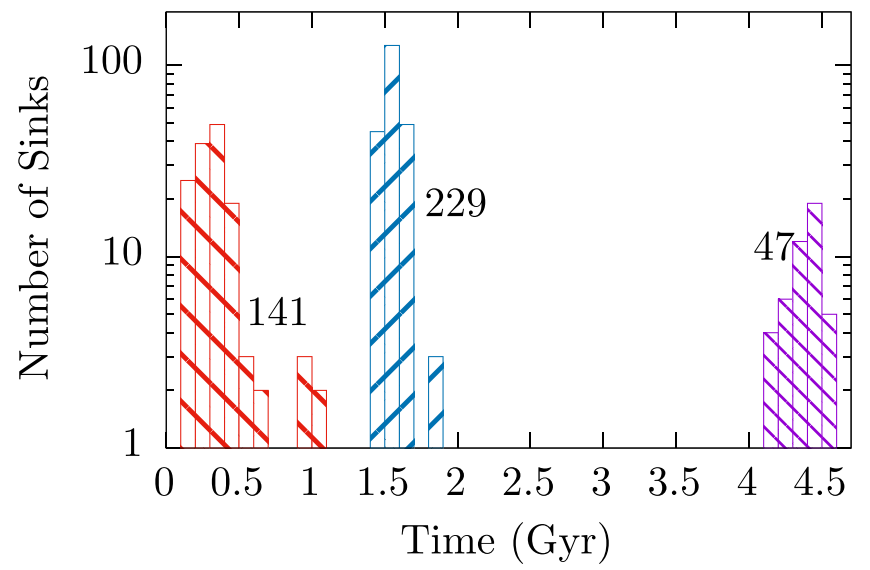

Figure 2. Globular star cluster formation in simulation MG2 clearly occurred in three distinct bursts; the numbers denote the total in each. The firstgeneration clusters (red) - roughly those formed before $1 \mathrm{Gyr}$-were produced in unstable regions of the progenitors before the initial interaction. The secondgeneration clusters (blue) — formed between $1 \mathrm{Gyr}$ and $2 \mathrm{Gyr}$-were produced during the first close pass of the colliding galaxies. Third-generation clusters (purple)—formed after $4 \mathrm{Gyr}$ - were produced during the second pass.

tidal fields. Sink particles were given unique integer identifiers during the simulation, generated sequentially by time of formation, starting at unity.

Star cluster formation occurred in three distinct episodes, as shown in Figure 2 (Li et al. 2005, 2006, 2007). Although the modeled starbursts are unlikely to be realistic in every detail, they do provide a qualitatively correct tidal environment modeled with more physical processes than previous work. We refer to those clusters that formed before the first passage, i.e., roughly before $1 \mathrm{Gyr}$, as first generation; they had IDs in the range 1-141. They formed in unstable regions in the progenitor galaxies. Second-generation clusters formed during the first interaction; significant formation activity is visible around $1.5 \mathrm{Gyr}$; their IDs were 142-370. A final formation episode occurred on the second passage, shortly after 4 Gyr; their IDs were 371-417. Unfortunately, there is very little dynamical history for these final clusters, therefore they are omitted from our analysis.

Figure 3 shows sink particles in the $X-Y$ plane of the simulation volume; first-generation sinks are colored red, second generation are blue, and third generation are purple. Four important periods of the simulation are shown:

1 Gyr: just before the first interaction, but after some star clusters have formed in initially unstable regions in the progenitors;

1.5 Gyr: during the first interaction;

4 Gyr: during the second lower velocity interaction, where the individual galaxies are still discernible;

5 Gyr: as the merger product virialized, shortly before the end of the simulation.

In these frames, the star clusters trace the areas of activity (such as the centers of the progenitor galaxies) fairly well because they form in the densest and most unstable regions.

The distribution of final galactocentric distances is shown in Figure 4, where it is clear that the first- and third-generation clusters are confined to the inner $7 \mathrm{kpc}$ by the end of the simulation, while the second-generation clusters can be found past $40 \mathrm{kpc}$.

\subsection{Tidal Histories}

At every snapshot we computed the tidal acceleration tensor at the position of each sink particle according to Equation (4). ${ }^{6}$ This produced a tensor-valued function representing the local tidal environment in the galactic inertial frame for every star cluster, sampled every $10 \mathrm{Myr}$. We refer to these functions as tidal histories. A selection is shown in Figure 5. These clusters were chosen to show a variety of features in the tidal accelerations.

Many of the first-generation star clusters formed in very dense and violent regions, and they tend to have strong irregular tidal accelerations. Sink 309 formed in a moderately active region, but subsequently moved into a much less perturbed periodic orbit. Sink 319 experienced a nearly constant tidal acceleration throughout its lifetime. Sink 305 moved slowly from an extensive tidal region into a compressive one, while sink 366 was basically an isolated cluster. One feature common to most sinks is a violent formation process, as evidenced by the initial spike in the tidal acceleration. Although some sinks formed in regions of extensive tidal forces, the sink formation criteria require gravitational binding of the gas (Li et al. 2005), guaranteeing that the parent clouds of the sink were indeed gravitationally bound.

This figure allows us to demonstrate that our approximation of neglecting the time derivatives at transitions between piecewise constant tidal forces (Section 3) is acceptable, because the energy input at each discontinuity in the tidal forcing is small compared to the total energy. This can be seen by comparing the initial total energy of our cluster, $E_{0}=-1 / 2$, to the typical changes in energy associated with the tidal forcing from our merger model. We have chosen units such that the eigenvalues of tidal acceleration can be directly compared to the changes in energy resulting. As can be seen in Figure 5, these changes remain small compared to $E_{0}$ at each point in time.

\section{Scaling $N$-body Simulations}

Having obtained a set of tidal acceleration curves, we conducted a series of numerical experiments with the extended version of KIRA, where we simulated the dynamics of star clusters with Equations (10) and (11) incorporated to model the effects of the external tidal field.

Because of computational constraints, we model a star cluster using a simulation containing fewer stars than typical real clusters $\left(1.6 \times 10^{4}\right.$ stars rather than the $10^{5-6}$ stars typical of globular clusters). The central issue, then, is how to scale the model to preserve the most important physical properties. Suppose that the real cluster can be characterized by the 4-tuple $\left(N^{\prime}, M^{\prime}, r_{\mathrm{h}}^{\prime}, \alpha_{1}^{\prime}\right)$ and the model system by $\left(N, M, r_{\mathrm{h}}, \alpha_{1}\right)$, representing the number of stars, mass, half-mass-radius, and the dominant eigenvalue of the tidal acceleration, respectively.

We quantify the strength of the tidal field on a cluster by the dimensionless parameter,

$$
\Gamma=\alpha_{1} \frac{r_{\mathrm{h}}^{3}}{G M} .
$$

\footnotetext{
6 To be more precise, we used a Plummer-softened form of the potential in order to maintain consistency with the form of the force law used during the merger simulations.
} 

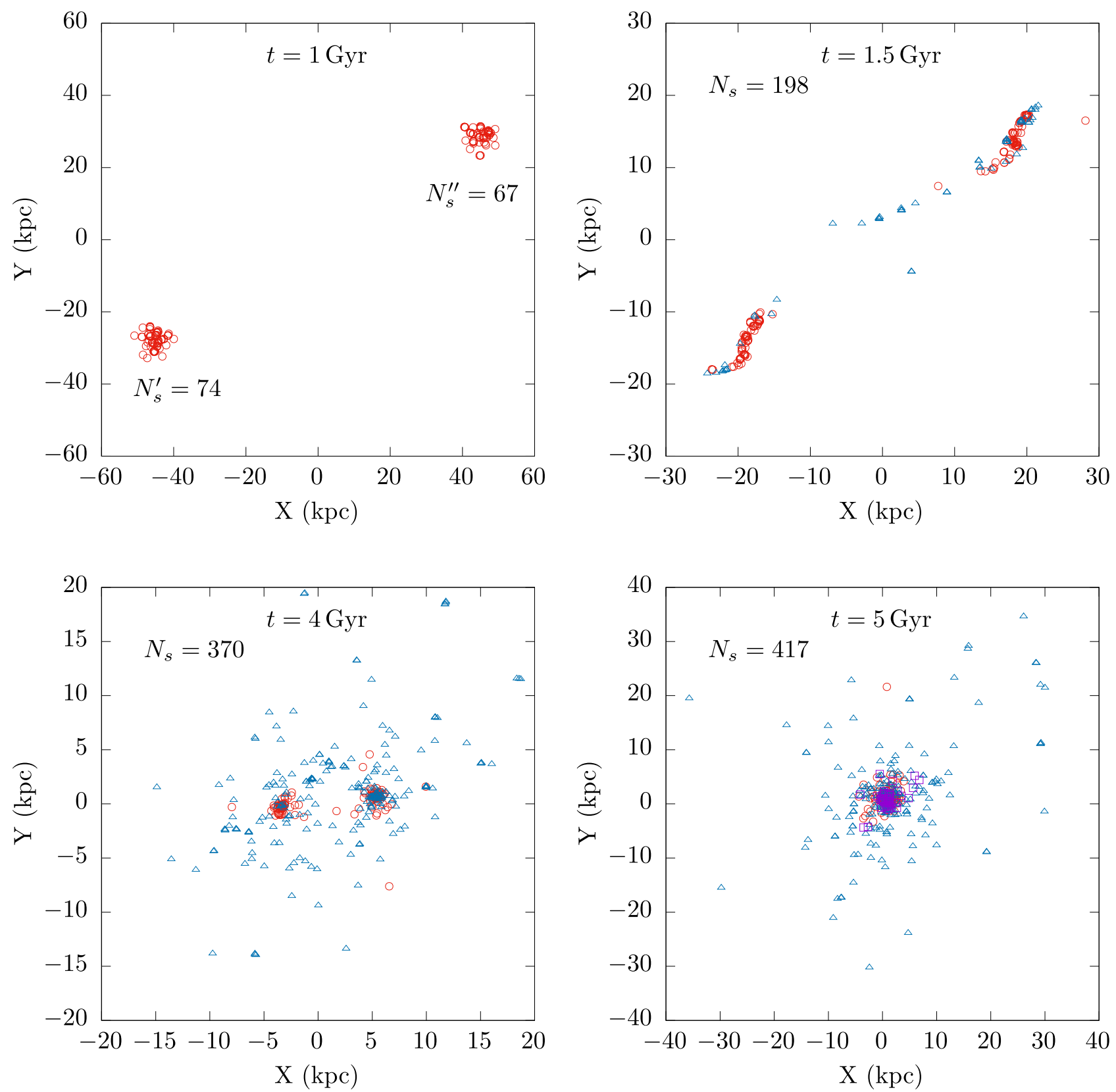

Figure 3. Star clusters forming during the course of the MG2 galaxy merger simulation. The first frame, at $1 \mathrm{Gyr}$, shows clusters that formed in the progenitors before the interaction. The snapshot at $1.5 \mathrm{Gyr}$ depicts clusters strewn along the arms during the first passage; this was a major cluster formation event. The frame at $4 \mathrm{Gyr}$ shows the interaction during the second passage; the centers of the individual galaxies are well represented by the density of star clusters. The final frame shows the virialized merger product at 5 Gyr. As in Figure 2, first-generation clusters are shown in red (circles), second generation in blue (triangles), and third generation in purple (squares).

We can now write

$$
\Gamma=\left(\frac{r_{\mathrm{h}}}{r_{\mathrm{t}}}\right)^{3}=\left(\frac{t_{\mathrm{dh}}}{t_{\mathrm{dt}}}\right)^{2}
$$

where $t_{\mathrm{dh}}$ and $t_{\mathrm{dt}}$ are defined by Equations (15) and (16), respectively.

Ideally, we would like to preserve all relevant physical scales, but this is impossible because different quantities scale differently with the number of stars $N$. In particular, we cannot preserve both relaxation time and dynamical time simultaneously (Aarseth \& Heggie 1998). In this situation, mass loss will manifest as stellar flow through the tidal boundary. The timescale for the loss of stars near the tidal boundary in the case of sudden changes in the tidal radius is the dynamical time at the tidal radius. Therefore we choose to preserve that dynamical time. We do this by setting

$$
\begin{gathered}
r_{\mathrm{h}}=\left(\frac{N}{N^{\prime}}\right)^{1 / 3} r_{\mathrm{h}}^{\prime}, \\
\alpha_{1}=\alpha_{1}^{\prime}, \\
\Gamma=\Gamma^{\prime} .
\end{gathered}
$$

The last equation implies

$$
\frac{r_{\mathrm{t}}}{r_{\mathrm{h}}}=\frac{r_{\mathrm{t}}^{\prime}}{r_{\mathrm{h}}^{\prime}} .
$$




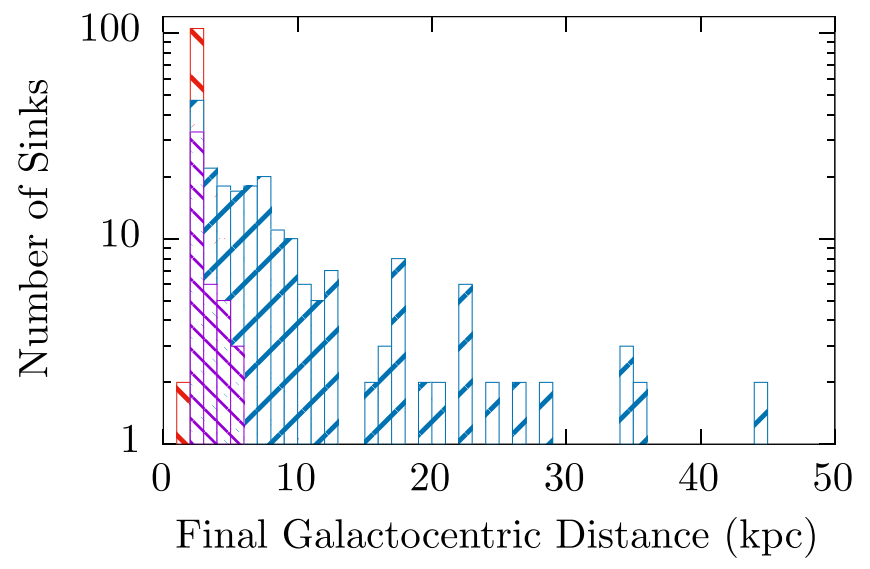

Figure 4. Distribution of the final galactocentric distance of star clusters formed in MG2. First-generation clusters (red) are confined to the inner $7 \mathrm{kpc}$, second generation (blue) are less concentrated, and third generation (purple) are again confined to the core of the merger product.

We note that this choice of scaling implies a constant-density cluster mass-radius relation, $r_{\mathrm{h}} \sim M^{1 / 3}$, and a constant mean particle mass, $M / N=M^{\prime} / N^{\prime}$.

We still have the freedom to choose the actual value of the dynamical time. To convert the physical units of the tidal acceleration tensor obtained from the galactic merger simulations into the dimensionless units of the $N$-body cluster simulations, we used the typical value for globular clusters of $t_{\mathrm{dv}}=1 \mathrm{Myr}$ (Portegies Zwart et al. 2001).

The results of simulations performed as described in this section are shown in Figure 6. We have performed several dozen such simulations, and these were used to calibrate the semianalytic model that we describe in Section 6. These simulations were also used to understand the accuracy of the model; this is shown in Figure 8.

\section{Mass-loss Model}

A full dynamical simulation of one of our star clusters with KIRA requires days or weeks of computational time. Considering that we are mainly interested in lifetimes and total mass loss and not in detailed dynamical histories, this approach is needlessly resource intensive. It is therefore desirable to obtain a simple analytic model for estimating mass loss, given a set of cluster parameters and tidal history.

Our models are fit to simulations that did not reach core collapse. However, this produces a negligible additional amount of mass loss because of the small volume occupied by the core (for example, see Figure 6 of Baumgardt \& Makino 2003, which shows no deviation from linear mass loss at the time of core collapse), and therefore we believe that this neglect does not significantly affect the quality of our model.

Dynamical effects of a time-variable tidal field can induce stellar mass loss through several underlying processes. We find it most useful to decompose the overall phenomenon into two distinct parts: internal two-body relaxation under the influence of the external tidal field, and changes in the Jacobi radius driven by that tidal field.

\subsection{Two-body Relaxation}

Even in isolation, a star cluster will undergo steady evaporation, as particles gain enough energy to escape, through two-body interactions. For clusters evolving in the external tidal field of their host galaxy, the mass-loss rate increases because the threshold for escape energy is lowered. It has been shown that for a broad range of values of $r_{\mathrm{h}} / r_{\mathrm{t}}$, mass loss is determined by the mean density within the tidal radius $\bar{\rho}_{\mathrm{t}}$ (e.g., Gieles \& Baumgardt 2008, and references therein),

$$
\frac{d N}{d t} \propto \sqrt{G \bar{\rho}_{\mathrm{t}}} \ln (\gamma N) .
$$

Considering particles of uniform mass $m$ and assuming that the tidal radius is proportional to the half-mass radius, we can substitute the definition of the tidal radius from Equation (14) to obtain

$$
\bar{\rho}_{\mathrm{t}} \propto \frac{N}{r_{\mathrm{t}}^{3}} \propto \frac{N \alpha_{1}}{G M} \propto \alpha_{1} .
$$

Substituting this into Equation (29), we find that the mass-loss rate due to enhanced evaporation from tidal acceleration acting on the two-body relaxation process is

$$
\frac{d M}{d t} \propto \alpha_{1}^{1 / 2} \ln (0.4 N),
$$

where we again use the Spitzer (1987) value $\gamma=0.4$.

In order to verify this piece of the model, we performed a set of simulations with a range of simple analytic tidal tensors, which were kept constant during the runs (note that these tensors are chosen just to test the numerical implementation of the model, and do not correspond to physical density distributions). The values were of the form

$$
\boldsymbol{T}=\left[\begin{array}{lll}
\alpha_{1} & & \\
& 0 & \\
& & 0
\end{array}\right],
$$

where the magnitudes $\alpha_{1} \in\{0.15625,0.3125,0.625,1.25,2.5$, $5\} \times 10^{-2} t_{\mathrm{dv}}^{-2}$ were chosen so as to cover a reasonable range of values seen from tidal histories described in Section 4. The initial conditions for these simulations were as described in Section 3. The given values of $\alpha_{1}$ imply $r_{\mathrm{t}} / r_{\mathrm{h}}=$ $\{11,8.9,7.0,5.6,4.4,3.5\}$, respectively. Figure 7 shows mass loss during the actual simulations together with the predictions of Equation (31). These predictions of slope are accurate for a large range of tidal field strengths, so long as the cluster remains bound and in gravitational equilibrium. As the field gains in strength and mass loss begins to deviate from the expected linear form, this model becomes less accurate. This can be observed in the last curve of Figure 7, when $\alpha_{1}=0.05 t_{\mathrm{dv}}^{-2}$, and the cluster loses mass in a superlinear fashion.

\subsection{Fluctuations in the Jacobi Radius}

The second element of our model relates to the changes in the Jacobi surface induced by a time-variable tidal field. Specifically, we aim to estimate the fractional mass loss $\Delta M / M$ for small variations of the Jacobi radius $\Delta r_{\mathrm{t}} / r_{\mathrm{t}}$. After a stronger tidal field establishes a smaller Jacobi radius, stars left outside are expected to escape on the local dynamical timescale.

We assume that near the tidal boundary the approximate radial mass dependence is

$$
M(r) \propto r^{a},
$$




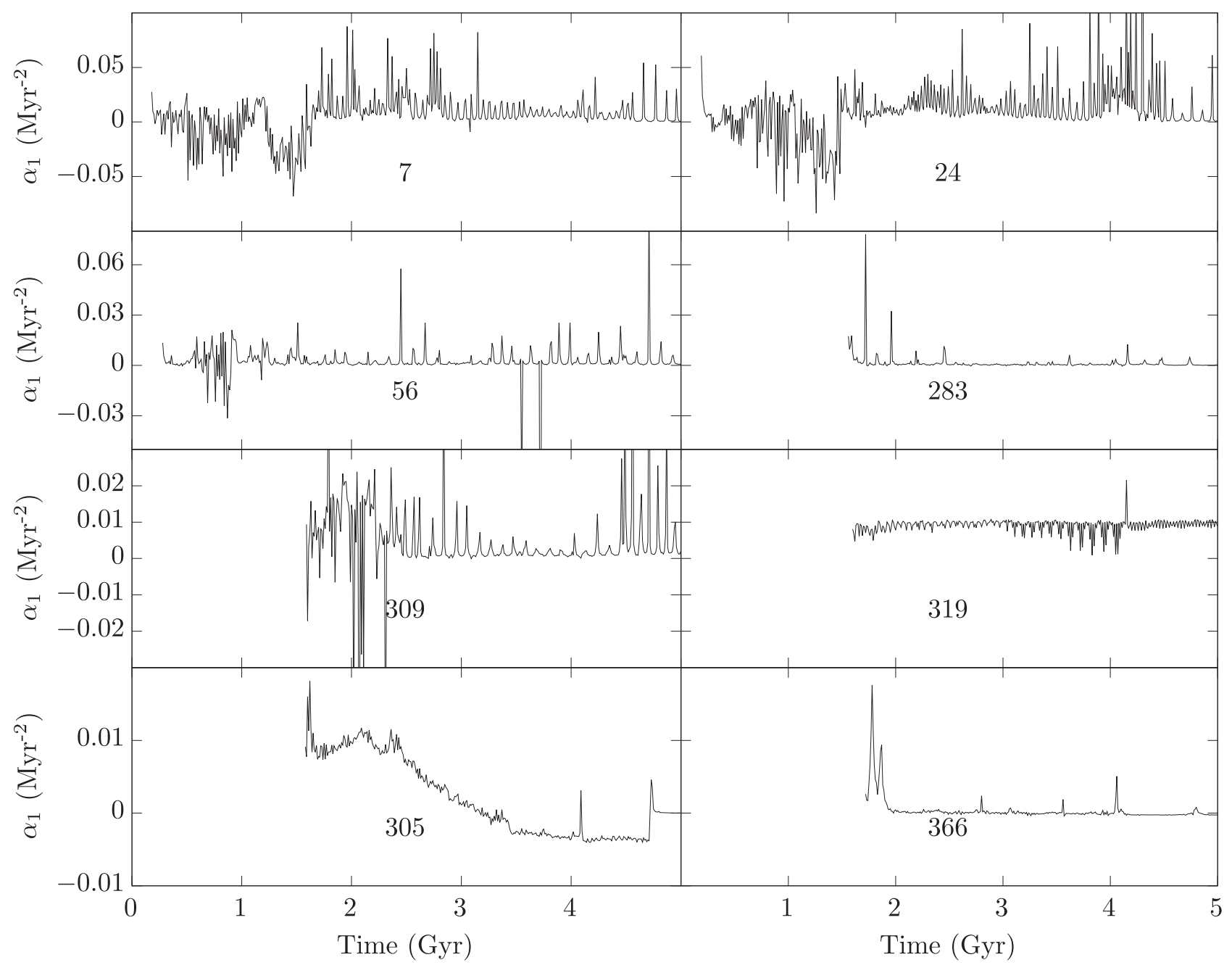

Figure 5. The dominant eigenvalue of the tidal acceleration is shown for a representative selection of sink particles from the galaxy merger simulation that we use. See Section 4.1 for a discussion of dynamical history revealed by these curves.

so that we can write the finite difference proportionality

$$
\frac{M\left(r_{\mathrm{t}}+\Delta r_{\mathrm{t}}\right)-M\left(r_{\mathrm{t}}\right)}{M\left(r_{\mathrm{t}}\right)} \simeq \frac{(\Delta M)_{\mathrm{tot}}}{M} \propto \frac{\Delta r_{\mathrm{t}}}{r_{\mathrm{t}}}
$$

where $(\Delta M)_{\text {tot }}$ the total amount of mass that would be lost due to a small decrease in the tidal radius $\Delta r_{\mathrm{t}}$ induced by an increasing tidal potential.

As discussed in Section 5, this mass will be lost on the local dynamical timescale $t_{\mathrm{dt}}$, so the mass-loss rate is $(\Delta M)_{\mathrm{tot}} / t_{\mathrm{dt}}$. Thus the amount of mass $\Delta M$ actually lost during a time $\Delta t$ since the change in the Jacobi radius occurred can then be found by substitution from Equation (34):

$$
\Delta M=\frac{(\Delta M)_{\mathrm{tot}}}{t_{\mathrm{dt}}} \Delta t \propto M \frac{\Delta r_{\mathrm{t}}}{r_{\mathrm{t}}} \frac{\Delta t}{t_{\mathrm{dt}}} .
$$

Differentiation of Equation (14) and algebraic rearrangement using the same equation give

$$
3 \frac{d r_{\mathrm{t}}}{r_{\mathrm{t}}}=\frac{d M}{M}-\frac{d \alpha_{1}}{\alpha_{1}} .
$$

Using the assumption of power-law radial mass dependence (Equation (33)), we can substitute $d M / M=a \frac{d r_{\mathrm{t}}}{r_{\mathrm{t}}}$, so

$$
(3-a) \frac{d r_{\mathrm{t}}}{r_{\mathrm{t}}}=-\frac{d \alpha_{1}}{\alpha_{1}} .
$$

Taking this in finite difference form, we find the proportionality

$$
\frac{\Delta r_{\mathrm{t}}}{r_{\mathrm{t}}} \propto \frac{\Delta \alpha_{1}}{\alpha_{1}} \text {. }
$$

Substituting this into the previous proportionality Equation (35) and algebraically rearranging gives

$$
\frac{\Delta M}{M} \propto \frac{\Delta \alpha_{1}}{\alpha_{1}}\left(\frac{\Delta t}{t_{\mathrm{dt}}}\right) .
$$

Combining Equations (14) and (16) shows that

$$
t_{\mathrm{dt}}=\alpha_{1}^{-1 / 2} \text {. }
$$

In final form, then, our estimate of mass loss due to a shrinking Jacobi surface over a time $\Delta t$ is

$$
\frac{\Delta M}{M} \propto \frac{\Delta \alpha_{1}}{\sqrt{\alpha_{1}}} \Delta t .
$$




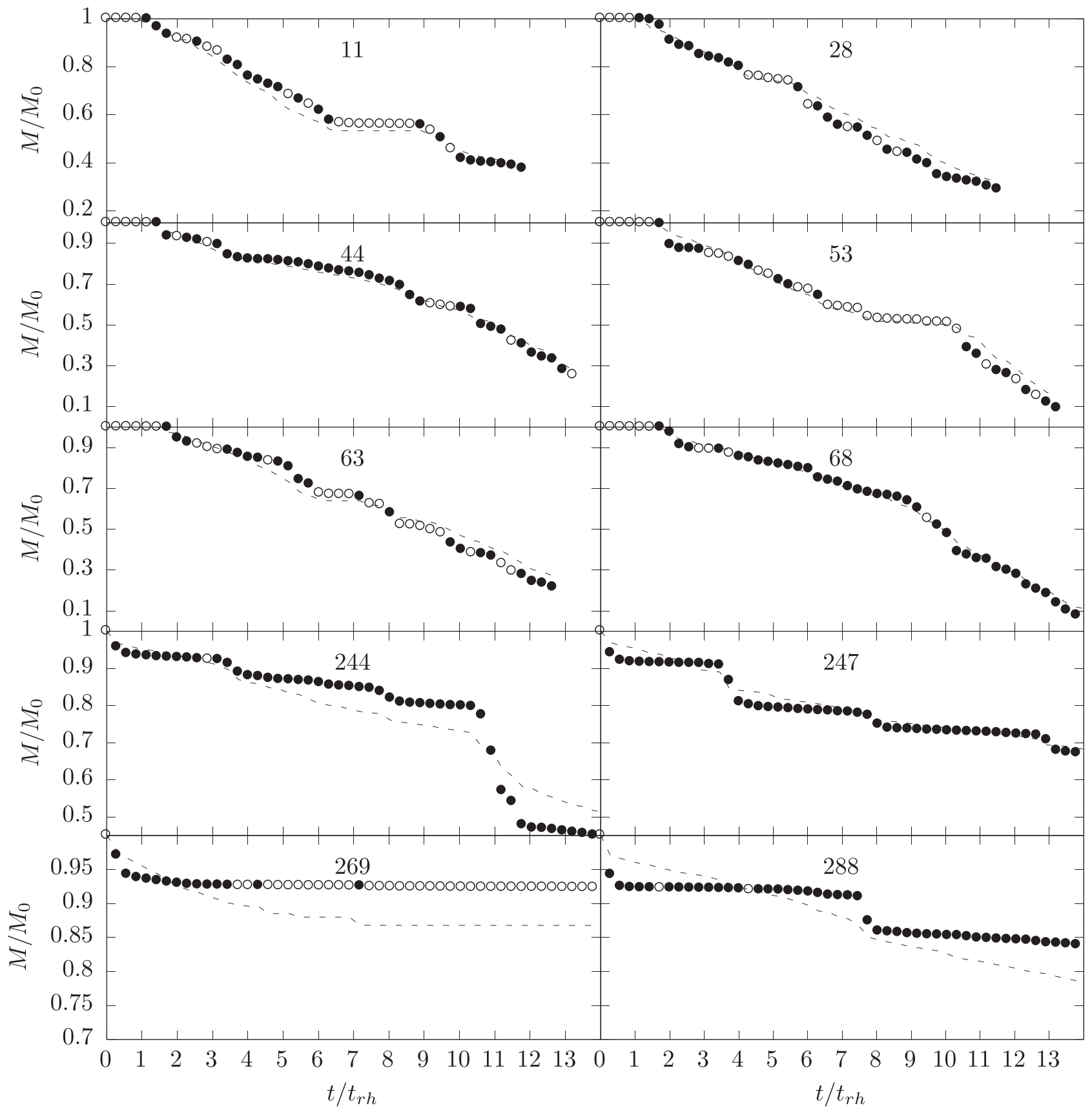

Figure 6. This figure shows mass-loss histories from a variety of environments using the full $N$-body simulations (circles). They are compared to our proposed massloss model (lines). Filled circles show when the dominant tidal mode is extensive, and open circles when it is compressive. Most of the clusters, as illustrated by Sinks $11,44,53,63$, and 68 , undergo fairly uniform mass loss and are tracked exceptionally well by the model. Some others experience short bursts of rapid mass loss, and these are typically not as well captured in the model; an example is sink 244 at around $t=4.5 t_{\mathrm{rh}}$.

Combining Equations (31) and (41) yields the expected mass loss due to enhanced evaporative pressure (first term) as well as contracting Jacobi radius (second term),

$$
\Delta M=A \sqrt{\alpha_{1}} \Delta t+B \frac{\Delta \alpha_{1}}{\sqrt{\alpha_{1}}} M \Delta t,
$$

where $A$ and $B$ are constants of proportionality.

Given the form of Equation (42), a few provisions must be made in order to handle all possible inputs. First, mass loss will only actually occur when $\alpha_{1} \geqslant 0$. More subtly, the second term is derived for cases of decreasing Jacobi radius, i.e., increasing tidal field strength. Our experiments have shown that clusters do not respond to negative, i.e., compressive, tides in any significant way, at least when they are under a few percent of internal binding energy. When iterating Equation (42) over the values of tidal acceleration, ${ }^{7}$ we therefore apply mass loss only during extensive periods, when $\alpha_{1} \geqslant 0$.

We further assume that the cluster comes into equilibrium with any applied tidal field, and that enhanced mass loss only occurs when that field grows stronger. Therefore we only engage the second term during periods of increasing tidal acceleration. Together these two criteria for mass loss can be

\footnotetext{
7 As mentioned before, the input is a time series of the diagonalized form of the tidal acceleration tensor.
} 


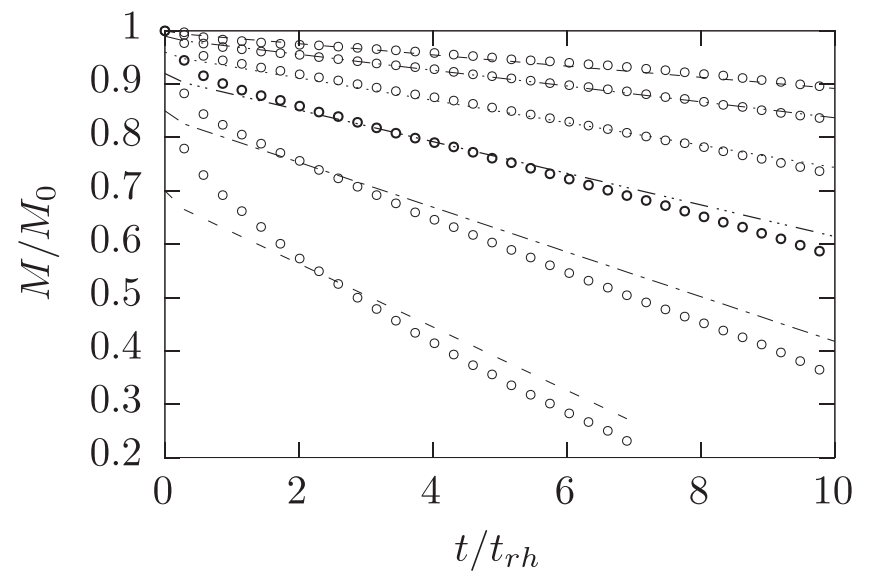

Figure 7. Mass loss under a constant tidal field with a range of values, from top to bottom, of $0.0015625 t_{\mathrm{dv}}^{-2}$ to $0.05 t_{\mathrm{dv}}^{-2}$. Open circles show the results of actual dynamical simulations, while lines are predictions from Equation (31). Since the relaxation piece of the model has no mechanism for the initial escape of stars outside of the newly imposed Jacobi radius, the solid curves have been lowered for easier comparison of the slopes.

expressed as

$$
\alpha_{1}>0 \text { and } \frac{d \alpha_{1}}{d t}>0 .
$$

With these caveats, Equation (42) becomes

$$
\Delta M=A \sqrt{\alpha_{1}} \Delta t+B\left(\frac{\alpha_{1}-\max \left(\alpha_{1}^{\prime}, 0\right)}{\sqrt{\alpha_{1}}}\right) M \Delta t,
$$

where $\alpha_{1}{ }^{\prime}$ is the value from the previous step and the numerator is recast for cases when the value of $\alpha_{1}$ changes sign from negative to positive. The max function ensures that only differences between positive tidal accelerations contribute to the mass loss.

In order to calibrate and validate the model, we performed detailed dynamical simulations with KIRA-enhanced with the prescription of Section 2-for a sample of a few dozen tidal histories obtained in Section 4 (including both compressive and extensive tides) and compared the resulting mass-loss curves to those predicted by Equation (42).

The $N$-body simulations were initialized with a Plummer model of 16384 particles, as described in Section 3. The tidal histories were scaled as described in Section 5 so that $t_{\mathrm{dv}}=1 \mathrm{Myr}$. This is ultimately an arbitrary choice that allows us to compare tidal histories by relative mass loss.

We now describe the procedure we used to estimate the constants $A$ and $B$, Equation (42), for the type of cluster we have simulated. Further work will be required to determine how universal these constants are. We varied the constants for multiple runs, gauging by eye the closeness of the model to the run. Since $A$ describes two-body relaxation under the influence of an external tidal field, we fixed the value of $A$ by considering a cluster in a constant tidal field, such as in Figure 7, and comparing the slope predicted by the model to that from the actual $N$-body simulation. It appeared that the value $A \approx 3.4 M_{\odot}$ worked well for a range of tidal field strengths. Having settled on the value for $A$, we compared the model to a series of simulations with time-varying Jacobi radii, and found $B \approx 0.135$. Our quoted values of $A$ and $B$ have a number of significant figures reflective of the level at which we could see variations in the goodness of fit. The panels in Figure 6 show

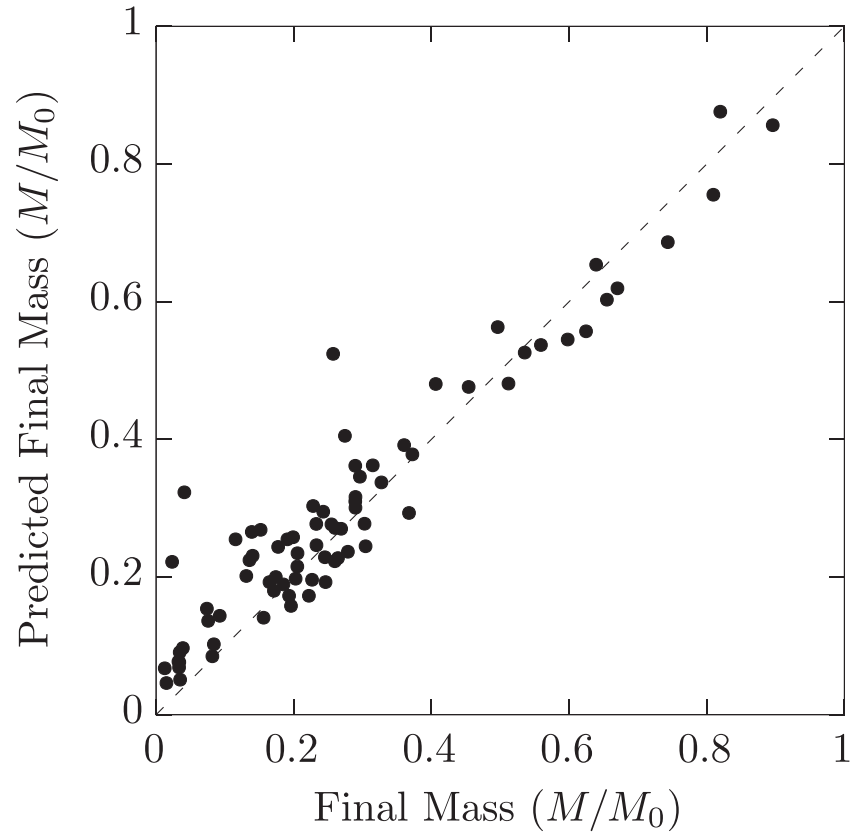

Figure 8. Final cluster masses for a series of full dynamical simulations are compared to the predicted masses from iterating Equation (42). The model tends to be more accurate for clusters with weaker external tidal fields, which lose less mass.

the comparison of the model with these values for a selection of $N$-body simulations.

To gain a more statistical view of the accuracy of the model, we also compared the mass of each cluster at the end of the simulation $^{8}$ to that predicted by the model at the same time; this is shown in Figure 8. There is more scatter for clusters that underwent more severe mass loss, and this is consistent with the analysis in Section 7. However, there are also more points in that region of the plot because sink particles were more likely to form in violent regions and thus suffer more severe mass loss during their early lives.

The figure, however, somewhat understates the accuracy of the model by only comparing the masses at the final state, by which point differences have accumulated. This is easily seen in Figure 9, where the model is fairly accurate for most of the run, but differences grow toward the end.

\section{Discussion and Summary}

The proposed model seems to capture the complex dynamical process of mass loss under arbitrary time-variable tidal fields fairly accurately; this is demonstrated by Figures 6 and 8. The savings in computational requirements allow us to perform the equivalent of millions of full dynamical simulations in minutes.

\subsection{Comparison with Related Work}

A few investigations have addressed questions similar to those investigated in this paper. Renaud et al. (2011) investigated a very similar scenario, sampling the tidal acceleration from a galactic merger simulation. We find that when the gravitational effects of dense regions of interstellar gas and newly formed star

\footnotetext{
8 The end of a simulation did not necessarily correspond to full dissolution or the end of tidal data. A few runs slowed down substantially due to pathological hard binaries and were terminated, a problem faced by all $\mathrm{N}$-body simulations.
} 
Tidal Acceleration (Sink 56)

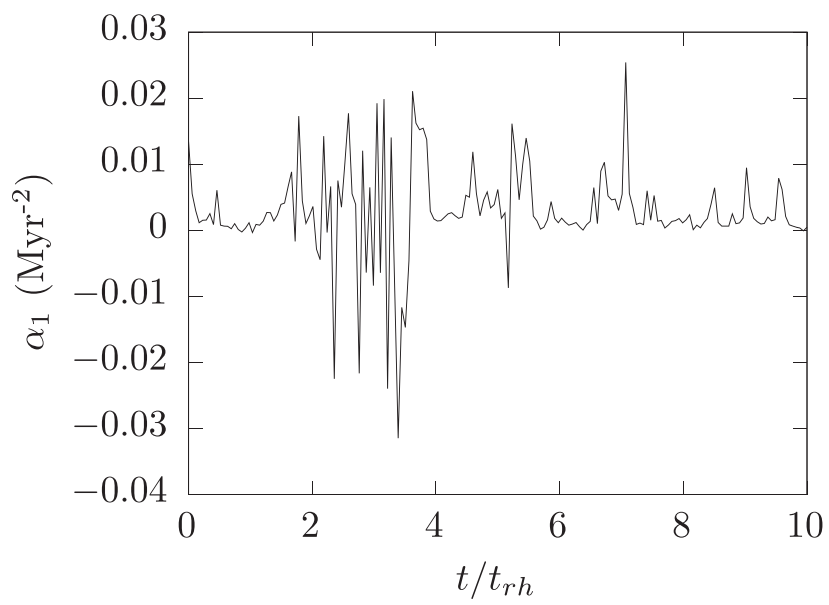

Mass Loss (Sink 56)

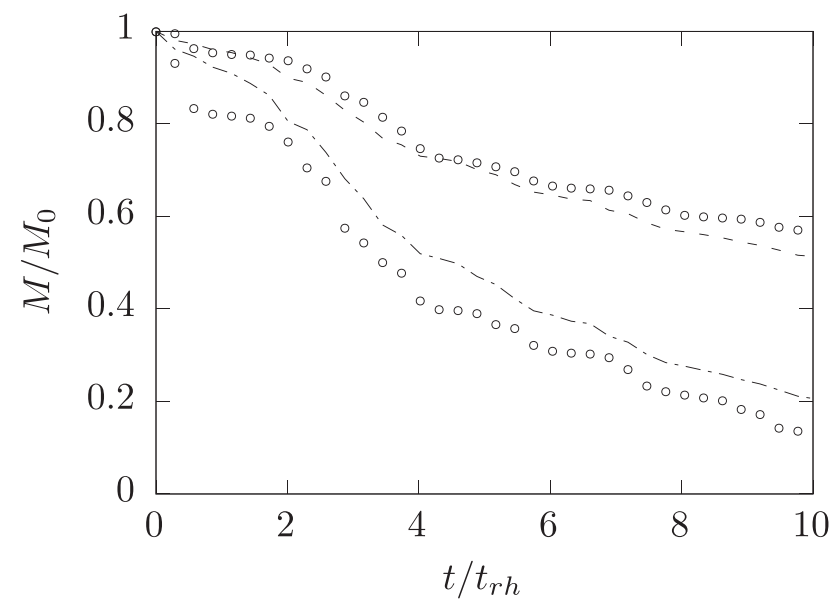

Tidal Acceleration (Sink 283)

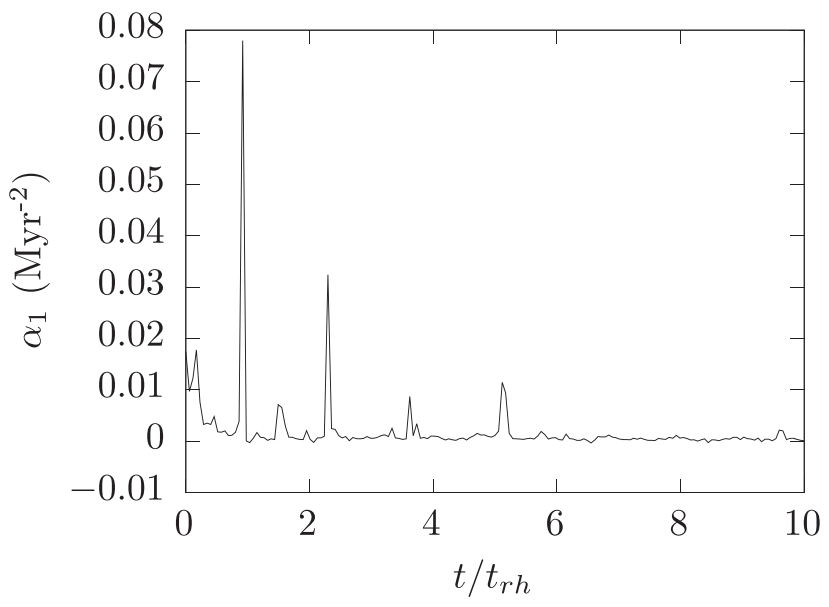

Mass Loss (Sink 283)

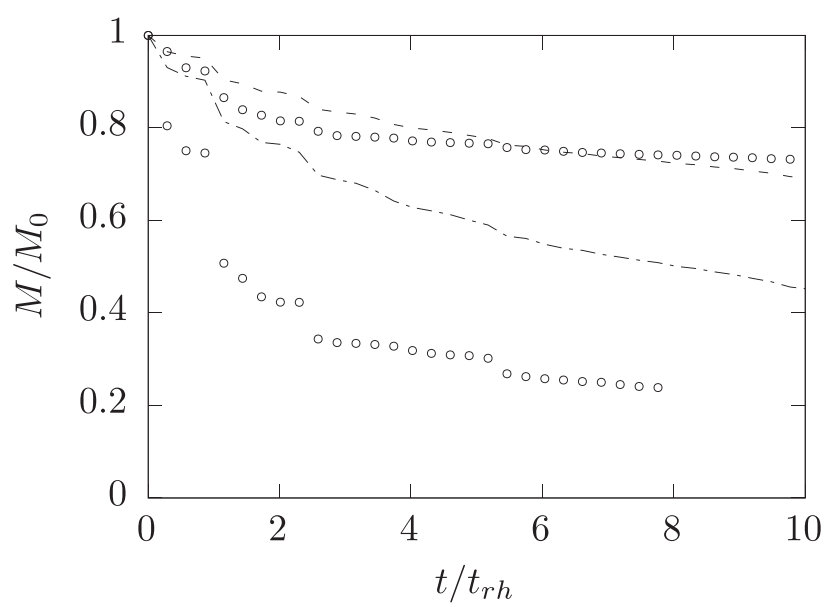

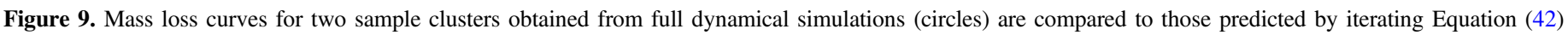

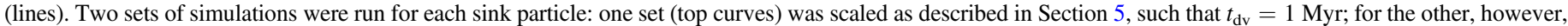

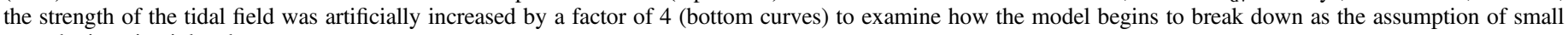
perturbations is violated.

clusters are taken into account, the result is a marked increase in the mass loss and dissolution rates of our model clusters. However, we focus in this work on a semianalytic approximation that will in future work allow for the results of many more tidal histories to be examined. Rieder et al. (2013) obtained their tidal accelerations from a cosmological model at much lower resolution and thus found correspondingly lower variations in the tidal field. They also did not propose a semianalytic approximation. We expect discontinuities of tidal histories, like those in Figure 5, to become more dramatic as the resolution of galactic simulations increases.

The conceptual approach of considering the changing tidal radius contrasts with the semianalytic approach proposed by Kruijssen et al. (2012), where tidal heating - that is, energy input due to the changing tidal field-is seen as the driving cause of mass loss. However, we argue that the role of tidal heating is secondary since we are able to account for mass loss without taking energy input into account.

\subsection{Limitations}

It is important to consider the model's limitations. One is that we did not vary the initial structure of the cluster under consideration to see whether the constants derived for the model might vary based on that. Another limitation is immediately apparent from Figure 6 at points where clusters experience rapid mass loss due to large spikes in the tidal acceleration. Examples of this are evident in the mass-loss curve of Sink 244 at $t \approx 4.5 t_{\mathrm{rh}}$ or Sink 288 at $t \approx 3.2 t_{\mathrm{rh}}$. In both cases, the model underestimates the effect of sudden contractions of the tidal radius probably because mass loss due to large fluctuations in the tidal radius is more sensitive to structural parameters, which we do not take into account. Furthermore, large fluctuations in the tidal radius will render the linearization in Equation (34) inaccurate.

Another unaccounted-for phenomenon occurs when a cluster substantially overfills its initial Jacobi radius. This is visible to various degrees with Sinks 53, 247, 269, and 288. For clusters initially underfilling their Jacobi radii, like 11 or 63 , the model accurately tracks the mass loss of full simulations.

We decided to investigate this phenomenon further and artificially increased the tidal field in order to prompt more drastic mass loss. Figure 9 shows two sink particles under the following setup. Each panel is dedicated to a cluster that was simulated with (a) its natural tidal history, and (b) one that was arbitrarily increased by a factor of 4 . For Sink 56, this increase causes a more 
rapid mass loss, and the model's agreement deteriorates somewhat as compared with the natural tidal history. The difference is most dramatic in the very beginning because a substantial mass fraction lies outside the tidal radius, something the model has no provision to handle. The situation is far more spectacular for Sink 283, which starts so far out of equilibrium that it lost $70 \%$ of its mass within one initial half-mass relaxation time.

The particular issues described in this section highlight two assumptions: (a) the tacit assumption of initial equilibrium, and (b) the explicit assumption that individual changes in the Jacobi radius be small. Cases where the model underperformed, as demonstrated in Figures 6 and 9, violated at least one of these assumptions. It might also be possible to better calibrate the constants in Equation (42), in particular $B$, which controls the reduction of mass at times of shrinking Jacobi radius.

Nevertheless, there is substantial agreement between the model and a wide range of tidal histories, and even in its current preliminary state, this allows for statistical studies that previously were computationally unfeasible. Ultimately, our approximation, which is based on models of a single cluster, will need to be refined with simulations of clusters with a wide range of structural parameters such as initial density profile in order to understand the dependence on them. In this study, we have taken the first step of investigating the mass loss resulting from various tidal histories acting on a single cluster.

We thank D. Kruijssen for useful discussions, and Y. Li for assisting with the use of her merger simulations. E.M. and S.M. acknowledge support from grant NSF grant AST-0959884. M.M.M.L. was partly supported by NSF grant AST-1109395 and the Humboldt Foundation, and thanks the Aspen Center for Physics, supported by NSF Grant PHY-1066293, for hospitality during some of the work on this paper. Support from grant $H S T$-AR-11780.01 is acknowledged. We thank the anonymous referee for a detailed report that resulted in a stronger paper.

\section{References}

Aarseth, S. J. 2003, Gravitational N-Body Simulations (Cambridge: Cambridge Univ. Press)

Aarseth, S. J., \& Heggie, D. C. 1998, MNRAS, 297, 794

Bate, M. R., Bonnell, I. A., \& Price, N. M. 1995, MNRAS, 277, 362

Baumgardt, H. 2001, MNRAS, 325, 1323
Baumgardt, H., \& Makino, J. 2003, MNRAS, 340, 227

Bertin, G., \& Varri, A. L. 2008, ApJ, 689, 1005

Binney, J., \& Tremaine, S. 2008, Galactic Dynamics (Princeton, NJ: Princeton Univ. Press)

Chernoff, D., Kochanik, C., \& Shapiro, S. 1986, ApJ, 309, 183

Elmegreen, B. G., \& Hunter, D. A. 2010, ApJ, 712, 604

Gaburov, E., Harfst, S., \& Portegies Zwart, S. F. 2009, NewA, 14, 630

Gieles, M., \& Baumgardt, H. 2008, MNRAS, 389, L28

Giersz, M., \& Heggie, D. C. 1994, MNRAS, 268, 257

Hartle, J. B. 2003, Gravity-An Introduction to Einstein's General Relativity (San Francisco, CA: Addison Wesley)

Heggie, D., \& Hut, P. 2003, The Gravitational Million-Body Problem (Cambridge: Cambridge Univ. Press)

Heggie, D. C., \& Mathieu, R. D. 1986, in The Use of Supercomputers in Stellar Dynamics, ed. P. Hut \& S. L. W. McMillan (Berlin: Springer)

Heggie, D. C., \& Ramamani, N. 1995, MNRAS, 272, 317

Kennicutt, R. C., Jr. 1998, ApJ, 498, 541

Kruijssen, J. M. D., Pelupessy, F. I., Lamers, H. J. G. L. M., et al. 2012, MNRAS, 421, 1927

Lee, H. M., \& Ostriker, J. P. 1987, ApJ, 322, 123

Li, Y., Haiman, Z., \& Mac Low, M.-M. 2007, ApJ, 663, 61

Li, Y., Mac Low, M.-M., \& Klessen, R. S. 2004, ApJL, 614, L29

Li, Y., Mac Low, M.-M., \& Klessen, R. S. 2005, ApJ, 626, 823

Li, Y., Mac Low, M.-M., \& Klessen, R. S. 2006, ApJ, 639, 879

Makino, J. 1991a, ApJ, 369, 200

Makino, J. 1991b, PASJ, 43, 859

Makino, J., Fukushige, T., Koga, M., \& Namura, K. 2003, PASJ, 55 1163

McMillan, S. L. W. 1986, in The Use of Supercomputers in Stellar Dynamics, ed. P. Hut \& S. L. W. McMillan (Berlin: Springer)

Portegies Zwart, S. F., McMillan, S. L. W., \& Gieles, M. 2010, ARA\&A, 48, 431

Portegies Zwart, S. F., McMillan, S. L. W., Hut, P., \& Makino, J. 2001, MNRAS, 321, 199

Renaud, F., Boily, C. M., Fleck, J.-J., Naab, T., \& Theis, Ch. 2008, MNRAS, 391, L98

Renaud, F., Bournaud, F., Kraljic, K., \& Duc, P.-A. 2014, MNRAS, 442, L33

Renaud, F., \& Gieles, M. 2013, MNRAS, 431, L83

Renaud, F., Gieles, M., \& Boily, C. M. 2011, MNRAS, 418, 759

Rieder, S., Ishiyama, T., Langelaan, P., et al. 2013, MNRAS, 436, 3695

Spitzer, L., Jr. 1987, Dynamical Evolution of Globular Clusters (Princeton, NJ: Princeton Univ. Press)

Springel, V., Yoshida, N., \& White, S. D. M. 2001, NewA, 6, 79

Varri, A. L., \& Bertin, G. 2009, ApJ, 703, 1911

Vesperini, E., \& Heggie, D. C. 1997, MNRAS, 289, 898

Weinberg, M. D. 1994a, AJ, 108, 1398

Weinberg, M. D. 1994b, AJ, 108, 1403

Weinberg, M. D. 1994c, AJ, 108, 1414

Whitmore, B. C., \& Schweizer, F. 1995, AJ, 109, 960

Whitmore, B. C., Zhang, Q., Leitherer, C., et al. 1999, AJ, 118, 1551 